\title{
Using Repetitive Control with Force Feedback to Reduce Impedance of Exoskeletons for Gait Training
}

\author{
Robert L. McGrath ${ }^{1}$, Student Member, IEEE, and Fabrizio Sergi ${ }^{1,2+}$, Member, IEEE
}

\begin{abstract}
Transparent interaction, or the reduction of human-robot interaction forces, is an important quality of gait training exoskeletons. In this paper, we investigate the feasibility of using a repetitive controller for reducing impedance of gait training exoskeletons using force feedback. We used a two-mass spring damper model system, and simulated the application of repetitive force controllers with the objective of reducing the end-point impedance of the distal mass. We designed and applied three repetitive controllers: a 1st order, a 2nd order designed for random signal period error, and a 2nd order designed for constant signal period error. We compared these three repetitive controllers subject to plant model parameter error, random signal period error, and constant signal period error. Numerical simulations under nominal conditions show that via repetitive force control, it is possible to reduce the endpoint impedance to the targeted magnitude and RMSE force below the limit achievable with force controllers while guaranteeing passivity. Furthermore, we established that the application of a 2 nd order repetitive controller designed for random period error is highly robust to random period error - exceeding the performance of the passive proportional controller up to $30 \%$ error of nominal frequency. Furthermore, this 2nd order repetitive controller designed for random period error maintains a $100 \%$ convergence rate through $60 \%$ plant parameter error.
\end{abstract}

\section{INTRODUCTION}

Transparent human-robot interaction, or the achievement of low interaction forces during unassisted movements, is a necessary quality of effective lower extremity exoskeletons. Commonly during gait training protocols there are phases of baseline and after-effect evaluation in which the exoskeleton is set to zero-torque mode and interaction forces are not desired. Force feedback is a simple method implemented on previous robotic platforms [1], [2], but has limitations due to the fact that the input of the plant does not change until error is already observed. Furthermore, stability of force-feedback system crucially depends on an accurate knowledge of the dynamics of the controlled system, knowledge that can be difficult to obtain in practice. In fact, a system using high control gains tuned to achieve high transparency performance may not be passive, and thus could be unstable during interaction with a percentage of the user population. Passivity is a desirable condition for the dynamics of a human-interacting exoskeleton, as it guarantees the stability of the robot when interacting with a range of environmental conditions [3],

\footnotetext{
This work was supported by the NSF-CBET-1638007

1 Department of Biomedical Engineering, University of Delaware, Newark, DE 19713, USA

2 Department of Mechanical Engineering, University of Delaware, Newark, DE 19713, USA

+ Corresponding author - fabs@udel.edu
}

[4]. The trade-off between stability and performance is particularly an issue on robotic platforms with high reflected inertia and therefore high end-point impedance [1]. As such, there is a need for novel controllers capable of reducing the robot's end-point impedance while remaining stable for a robust range of conditions such as those encountered during human-robot interaction.

In previous Bowden cable driven exoskeleton work, iterative learning control has been utilized to learn periodic cable tensioning patterns for the gait cycle [5]. The implementation of this learning controller was possible due to the cable slacking in the swing phase of the gait cycle, creating a reset point between periods. However, not all exoskeletons have this possibility, and in most cases the interaction forces are continuous signals with the same periodicity as the resulting motion.

The repetitive controller ( $\mathrm{RC}$ ), intended for periodic and continuous operation in the time domain, could be a feasible option for gait training exoskeletons during continuous operation [6]. RC is a feedforward control method for reducing error of the current task cycle based on the error observed in the previous task cycles, and is suitable for continuous operations. In robotics, RCs have been applied to trajectory control [7] and we are not aware of any applications of these controllers in force control.

RCs require some knowledge of the dynamics of the system they are applied to, and have traditionally been applied to systems with highly regular periodicity. In gait training exoskeletons, the dynamics of the coupled system are usually not known with great accuracy due to betweensubject variability. Also, stride-by-stride variation in gait spatiotemporal parameters is on the order of 3-5\% [8]. There are formulations of RCs (high-order controllers) which combine information measured on multiple previous cycles to improve robustness input period error [9], [10]. However, the suitability of these controller to be used for gait training exoskeletons has yet to be established.

In this work, we present formulations for RCs that use force feedback to reduce the end-point impedance of a gait training exoskeleton. We test the performance of these RCs via simulations applied to a two-mass spring damper system classically used to study controllers for human-interacting robots under force control. We first simulate behavior in a case where we assume perfect knowledge of system parameters and perfect periodicity of subject inputs. Then, we conduct simulations in non-ideal conditions to assess the robustness of different formulations of the controller to variations in parameters of the system dynamics, and to 
variations in the period of the inputs that would be applied by the subject.

\section{METHODS}

\section{A. Mechanical System}

We use a two-mass model classically studied in force control for human-interacting robots [3], [4] to evaluate the behavior of the RCs under force feedback. The mechanical system considered in this paper, shown in Fig. 1, consists of two masses connected by a spring and damper in parallel. In the general case of a robotic exoskeleton, the proximal $M_{1}$ and distal $M_{2}$ masses are representative of the motor rotor and exoskeleton leg, respectively. The spring $K$ and damper $B$ in parallel are representative of the geared transmission dynamics.

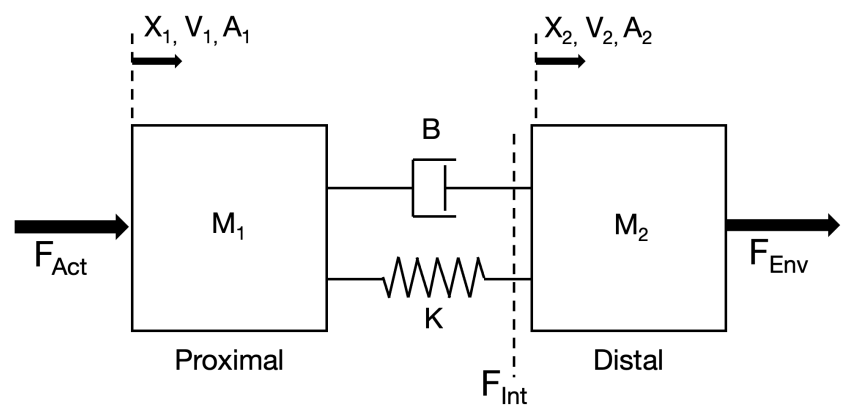

Fig. 1: Mechanical system of interest

The proximal and distal masses each have a position, velocity and acceleration as defined in Fig. 1. The distal mass interacts with an environment modeled as a velocity source $V_{2}$, as might theoretically be imposed by a human participant, with resulting environmental force $F_{E n v}$. The proximal mass has an applied control action motor force $F_{\text {Act }}$ as specified by controller architecture explained in the following sections. The interaction force $F_{\text {Int }}$ is the force that can be measured at the spring and damper, arranged in parallel, between the two masses.

\section{B. Passive Impedance Controller}

We are utilizing impedance control since it allows us to directly specify the desired dynamics we wish to display to the environment through a target impedance transfer function given an environmental velocity [4]. The capability and goal of the proposed impedance controller, shown in Fig. 2, is the reduction of end-point impedance below that of the distal mass alone. As such, the target impedance is given as:

$$
Z_{Q}(s)=Q \cdot M_{2} \cdot s
$$

In this expression, the target impedance is defined as a fraction $Q$ of the physical impedance of the distal mass. $Q$ ranges between 0 and 1 if the goal is to reduce the displayed impedance below the physical value of the distal mass. It is worth noting that a proportional force controller which aims to obtain an impedance in the form of equation 1 with $Q<1$ will not be passive [3]. The desired environmental force $F_{E n v_{D e s}}$ is derived by passing the prescribed distal

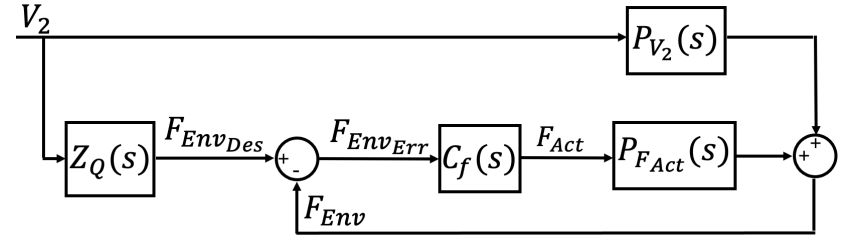

Fig. 2: Schematic of the impedance controller.

velocity $V_{2}$ through this transfer function block of the desired impedance $Z_{Q}(s)$.

The control action motor force $F_{A c t}$ applied to the proximal mass is obtained by taking the error between the measured environmental force $F_{E n v}$ and the desired environmental force $F_{E n v_{D e s}}$ and passing it through the control block transfer function $C_{f}(s)$. We are requiring the controller to meet the condition of passivity, to ensure stability when interacting with a range of environmental conditions [4]. As such, the control block transfer function $C_{f}(s)$ is required to satisfy the passivity requirement of:

$$
\operatorname{Re}\left\{Z_{E n v}(j w)\right\}=\operatorname{Re}\left\{\frac{F_{E n v}(j \omega)}{V_{2}(j w)}\right\} \geq 0 \quad \forall \omega \in \mathbb{R}
$$

This leads to the following expression for the passive upper limit on the proportional gain $K_{P}$ :

$$
C_{f}(s)=K_{P} \leq \frac{M_{1}}{M_{2}(1-Q)}
$$

The equation relating the environmental force $F_{E n v}$ to the distal velocity $V_{2}$ and control action motor force $F_{A c t}$ and plant transfer functions is given as:

$$
F_{E n v}=V_{2} \cdot P_{V_{2}}(s)+F_{A c t} \cdot P_{F_{A c t}}(s)
$$

Where the plant transfer functions $P_{V_{2}}(s)$ and $P_{F_{A c t}}(s)$ take the form:

$$
\begin{gathered}
P_{V_{2}}(s)=\frac{\left(M_{1} \cdot M_{2}\right) s^{3}+B\left(M_{1}+M_{2}\right) s^{2}+K\left(M_{1}+M_{2}\right) s}{M_{1} s^{2}+B s+K} \\
P_{F_{A c t}}(s)=\frac{B s+K}{M_{1} s^{2}+B s+K}
\end{gathered}
$$

Although the expressions for the transfer functions are derived in the s-domain, the transfer functions are simulated in the z-domain in the following sections using a zero order hold $\left(T_{s}=0.001 s\right)$

\section{Repetitive Force Control}

In an $\mathrm{RC}$, the error of the previous cycle is applied through a feed-forward system to compensate for the anticipated error of the current cycle. The general form of the first order RC [11] is as follows:

$$
U(z)=z^{-p} I(z) H(z)[U(z)+F(z) E(z)]
$$

The RC is designed and implemented in the $z$ domain. The controller's output $U(z)$ and error $E(z)$, operated on by a compensator $F(z)$, from the previous cycle (given delay $z^{-p}$ ) are combined to produce the output at the current cycle. The interpolator $I(z)$ is necessary if the number of time steps in 
a cycle $p$ is not an integer and the zero phase low-pass filter $H(z)$ can be applied if a frequency cutoff of the learning process is necessary.

The transfer function form of the general first order RC, converting the error input $E(z)$ to control output $U(z)$, is:

$$
R_{1}(z)=\frac{F(z) I(z) H(z)}{z^{-p}-I(z) H(z)}
$$

Given the context of simulation of this work, the interpolator $I(z)$ has been set to unity, as the capability of specifying any nominal frequency is not necessary in simulations. This yields the following simplified transfer function for a first order RC:

$$
R_{1}(z)=\frac{F(z) H(z)}{z^{p}-H(z)}
$$

It is known that a high-order RC can provide robustness to signal period error through the widening of the notches in the sensitivity transfer function [10], [12]-[14]. The sensitivity transfer function describes the influence of the command signal on the error signal and is defined as [15]:

$$
S(z)=\frac{1}{R(z) \cdot G(z)}
$$

The closed loop transfer function $G(z)$ is defined as:

$$
G(z)=\frac{C_{f} P_{F_{A c t}}(z)}{1+C_{f} P_{F_{A c t}}(z)}
$$

As such, we implemented an additional controller, a 2nd order RC, with generic transfer function form:

$$
R_{2}(z)=\frac{\phi F(z) H(z)\left[\alpha_{1} z^{p}+\alpha_{2}\right]}{z^{2 p}-H(z)\left[\alpha_{1} z^{p}+\alpha_{2}\right]}
$$

The parameter $\phi$ is the learning gain, and parameters $\alpha_{1,2}$ provide weights to the compensatory action resulting from the previous two cycles. These parameters are constrained such that:

$$
\begin{gathered}
0<\phi<1 \\
\alpha_{1}+\alpha_{2}=1
\end{gathered}
$$

For this work, we have implemented plug-in type RCs [15], [16], as shown in Fig. 3, where the RC transfer function is located in parallel to a standard feedback loop.

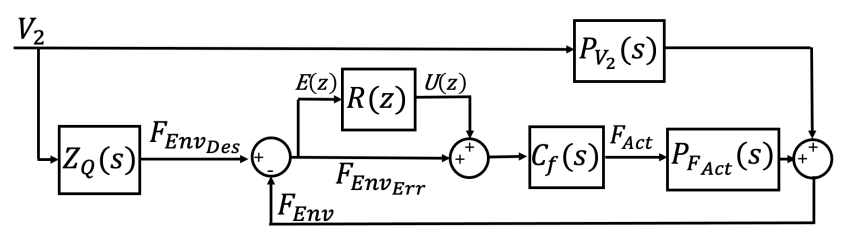

Fig. 3: Schematic of the impedance controller with applied plug-in type repetitive control.

We opted for this form of controller, as opposed to the cascade-type $\mathrm{RC}$, because the plug-in type could be conveniently enabled or disabled without disruptive transient of force error. This capability was deemed to be essential for safety-critical applications such as human-robot interaction.

\section{Repetitive Controller Tuning}

For these RCs, the compensator, a finite impulse response digital filter, is specified by two design terms $m \& n$, where the compensator has $m$ zeros and $n-m$ poles. We know that the closed-loop system with added RC (plug-in or cascade) and zero phase low-pass filter is stable if the following expression holds:

$$
M_{H F G}(\omega)=\left|H\left(\omega^{*}\right)\left(\frac{1+\left(1-F\left(\omega^{*}\right)\right) C_{f} P_{F_{A c t}}\left(\omega^{*}\right)}{1+C_{f} P_{F_{A c t}}\left(\omega^{*}\right)}\right)\right|<1
$$

where $\omega^{*}=e^{i \omega T_{s}}$

To determine a suitable compensator, the following expression is minimized [17] over the frequency range of interest utilizing fmincon in MATLAB (MathWorks Inc, Natick, MA):

$$
J=\max \left(\left|1-F\left(e^{i \omega T_{s}}\right) G\left(e^{i \omega T_{s}}\right)\right|\right)
$$

Two sets of 2 nd order RC weight parameters were taken from previous work [12]. These sets of parameters $\alpha_{1,2}$ were designed specifically for accommodating random period error (RPE) and constant period error (CPE) and assigned as $[2 / 3,1 / 3]$ and $[2,-1]$, respectively. The learning gain $\phi$ for both 2 nd order RCs leading to convergence was determined empirically to be 0.5 .

\section{E. Nominal Performance Evaluation}

All controller simulations were implemented via Simulink models with an environmental velocity signal obtained as the time integral of a unit magnitude acceleration signal, sampled at a specified interval $\left(T_{s}=0.001 \mathrm{~s}\right)$. Each nominal simulation began with 5 seconds of passive proportional control (PPC) followed by the plug-in type RC engaged for 30 seconds.

There are two primary assessment metrics of performance: the first is the root mean squared $F_{E n v_{E r r}}$ (RMSFE) for each cycle of the source velocity signal (nominal value: $1 \mathrm{~Hz}$ ). The second is the end-point impedance $Z_{E n v}$ over a range of excitation frequencies. The end-point impedance was tested at a set of frequencies that did not require an interpolator in order to be implemented.

\section{F. Robustness Analyses}

1) Model Parameter Error: The compensator optimization process, based on a model of the plant transfer function $P_{F_{A c t}}(s)$ and the passive control block $C_{f}(s)$, determines the stability of the RCs. However, in practical implementation of the RCs, there will exist error in the plant transfer function estimate relative to the actual physical plant. As such, it was important to asses the stability of the RC when there was error present in the modeled transfer function. In order to assess the robustness of the RC to model error; we assessed the convergence of the controllers when the compensator was designed with an ideal plant model and was applied to plant models with various magnitude of error applied to the individual parameters (i.e., $B, K, M_{1}, M_{2}$ ).

For each of the three RCs, we ran one hundred simulation repetitions for ten values of parameter error (i.e., $0 \%, 10 \%$, $\ldots, 90 \%)$. As such, if a nominal value was $10 \mathrm{~kg}$ and the 
parameter error was equal to $10 \%$, the value simulated in each of the 100 repetitions was randomly sampled from a uniform distribution with margins $[9,11] \mathrm{kg}$. Each simulation started with ten seconds of PPC followed by 490 seconds of plug-in type RC. The convergence of each simulation was assessed by the relative magnitudes of the RMSFE of early $\mathrm{RC}$ (mean of cycles 11-15) and late RC (mean of the last 10 cycles). If the late RMSFE values was less than the early RMSFE value, the simulation was considered to converge.

2) Source Signal Random Period Error: The controller is subject to the imposed environmental velocity source, modeled as a sinusoidal waveform. The period of this waveform is analogous to the period of the gait cycle during walking in a lower extremity exoskeleton. In human walking, the gait cycle period is subject to random errors, cycle to cycle. To assess the robustness of the RCs subject to RPE as seen in walking we assessed the RMSFE of the controllers when the period of the input velocity signal varied randomly with a uniform distribution.

For each simulation of the RPE analysis, the period of each individual cycle of the applied sinusoid was drawn from a uniform distribution with zero mean and variable half width. The half width was changed in different runs of the simulation, and ranged between $0 \mathrm{~Hz}$ to $0.90 \mathrm{~Hz}$ (in increments for $0.01 \mathrm{~Hz}$ ). The period of each individual cycle of the applied sinusoid was drawn from a uniform random distribution specified by the half width value. Each simulation began with 10 seconds of PPC followed by 190 seconds of plug-in type RC. The RMSFE of each controller was assessed: for PPC (mean of cycles 2-10) and for the RCs (mean of last 100 cycles).

3) Source Signal Constant Period Error: The period of the gait cycle in human walking is subject to CPE as might be observed during sustained gait speed changes. In order to assess the robustness of the RCs to CPE, we assessed the performance of the controllers when the gait cycle period was a consistent non-nominal value for the duration of the simulation. This is a worst-case scenario to assess the robustness of an RC to a discrepancy between the perturbation period and the period assumed by the RC. In practice, $\mathrm{RC}$ could be reset after a number of cycles, and the period of their action $p$ can be updated given measurements of the perturbation period.

We assessed RMSFE at a range of 51 different environmental velocity frequencies (i.e., $0.75-1.25 \mathrm{~Hz}$ ), with a constant spacing of $0.01 \mathrm{~Hz}$. Each simulation, at a specified environmental velocity frequency, began with 10 seconds of PPC followed by 90 seconds of plug-in type RC. The RMSFE of each controller was assessed: for PPC (mean of cycles 2-10) and for the RCs (mean of last 5 cycles).

\section{RESULTS}

\section{A. Repetitive Controller Tuning}

Prior to performing simulations, the compensator was designed according to the specified default set of parameters. This default set of parameters is given in Table I.
TABLE I: The default set of simulation parameters

\begin{tabular}{|c|l|l|}
\hline Parameter & Value & Description \\
\hline$M_{1}$ & $10 \mathrm{~kg}$ & Proximal mass \\
\hline$M_{2}$ & $5 \mathrm{~kg}$ & Distal mass \\
\hline$K$ & $200 \mathrm{~N} / \mathrm{m}$ & Spring constant \\
\hline$B$ & $20 \mathrm{Ns} / \mathrm{m}$ & Damping constant \\
\hline$Q$ & 0.10 & Desired fraction of distal mass \\
\hline$K_{P}$ & 2.2 & Passive proportional control gain \\
\hline$p$ & 1000 & Period in time steps \\
\hline$T_{s}$ & $0.001 \mathrm{~s}$ & Sample interval \\
\hline$f_{v}$ & $1 \mathrm{~Hz}$ & Velocity source frequency \\
\hline$A_{v}$ & $1 /(2 \pi) \mathrm{m} / \mathrm{s}^{2}$ & Velocity source amplitude \\
\hline$m$ & 9 & Compensator design term \#1 \\
\hline$n$ & 2 & Compensator design term \#2 \\
\hline$\phi$ & 0.5 & Learning gain \\
\hline$\alpha_{1,2}$ & {$[2 / 3,1 / 3] \&[2,-1]$} & RPE \& CPE RC weights \\
\hline
\end{tabular}
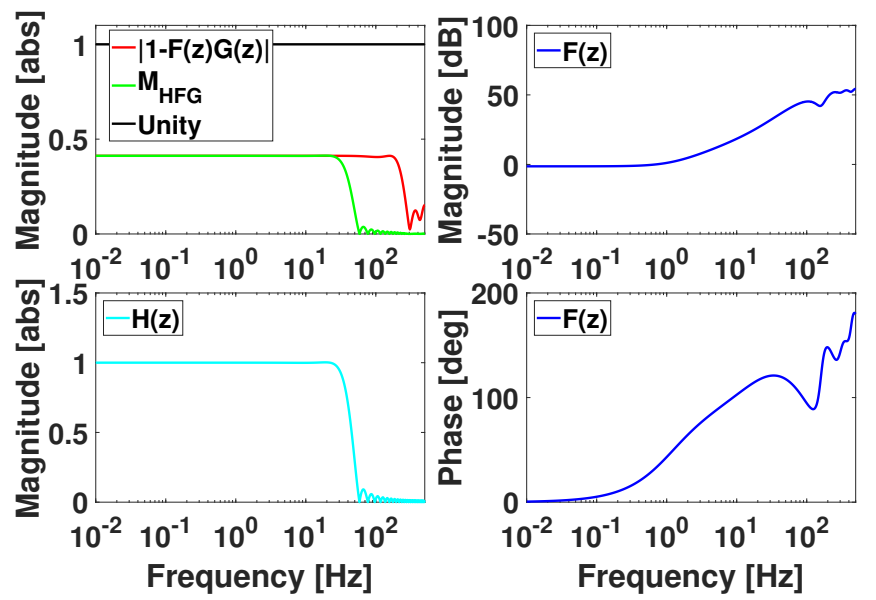

Fig. 4: (Top left) Asymptotic stability of the compensator when coupled with the nominal plant and zero-phase low pass filter, (bottom left) the magnitude of the low pass filter, and (top right) the magnitude and (bottom right) phase of the compensator.

It is important to note that we designate the value of $Q$ to be 0.10 such that our target end-point impedance is $10 \%$ of the natural impedance of the distal mass.

In Fig. 4, the optimized compensator is shown and the resulting asymptotic stability obtained when coupling the compensator with the nominal plant is apparent.

In Fig. 5 are the sensitivity magnitude plots of the four controllers. These describe the influence of the controller design on disturbance signals, such that a lower sensitivity, particularly in close proximity to the nominal frequency, is desirable.

\section{B. Nominal Performance}

The nominal simulations for plug-in type 1st order $\mathrm{RC}$, 2nd order RC designed for CPE, and 2nd order RC designed for RPE applied to the passive impedance controller are shown in Figs. 6-8, respectively. As can be seen, the PPC quickly achieves steady state, after which, the RCs are applied and achieve a new steady state after several cycles of learning. The 1st order RC reduces the RMSFE to below $5 \%$ of the original passive control RMSFE after 12 cycles of 


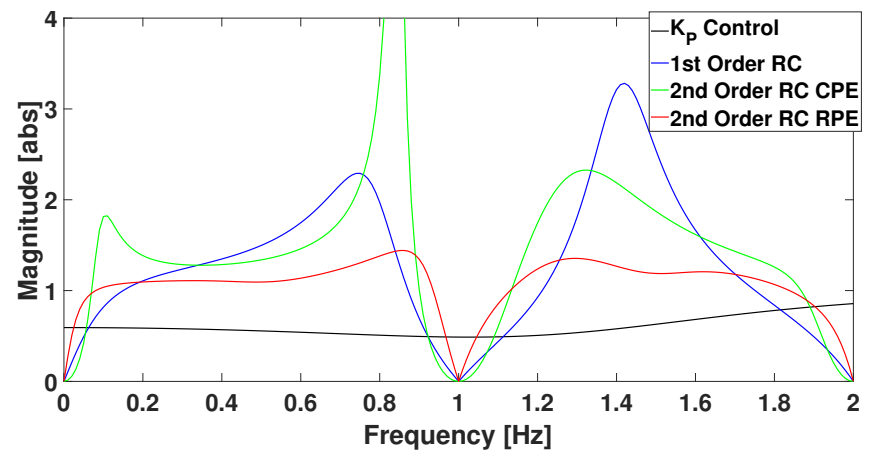

Fig. 5: Magnitude plots for the sensitivity transfer functions of the four controllers.

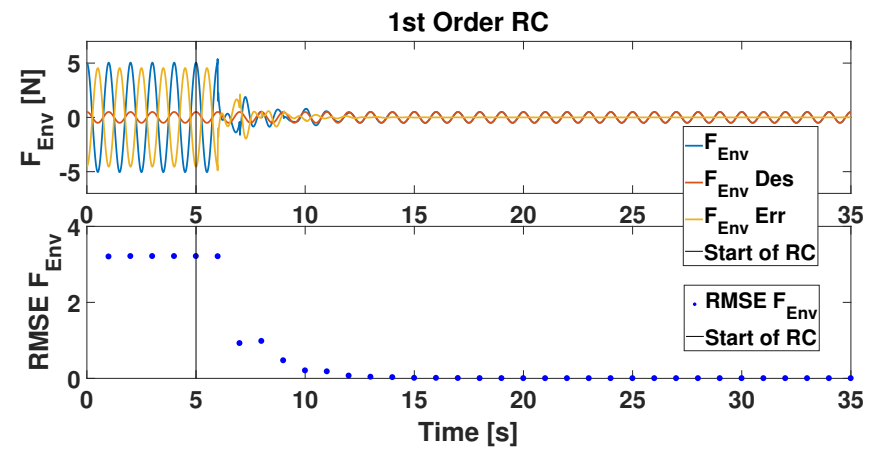

Fig. 6: (Top) example simulation of the plug-in type 1st order RC applied after 5 seconds of passive proportional control. (Bottom) the RMSE of $F_{E n v}$ for each cycle.

learning, where as the 2nd order RC designed for CPE and RPE require 37 and 18 cycles, respectively, of learning.

Shown in Fig. 9 are the impedance phase and magnitude of each controller. The magnitude of the passive impedance controller aligned with the theoretical impedance of $M_{2}$, indicating the same end-point impedance. Most importantly, the impedance of the impedance controller with applied $\mathrm{RC}$ at specified frequencies were close to the theoretical magnitude $Z_{Q}(s)$ indicating the successful reduction in endpoint impedance by the 1 st order RC controller. For all evaluated controllers, the impedance phase was within the range $[-90,90]$ deg, indicating passivity of the controlled system.

\section{Robustness Analyses}

1) Model Parameter Error: Depicted in Fig. 10, all controllers performed with $100 \%$ of simulations converging at nominal parameter values $(0 \%$ error). The 1 st order RC performed with $100 \%$ of simulation converging through $20 \%$ parameter error and moderately decreasing rates until just under $70 \%$ at $60 \%$ parameter error. The best performance was by the RPE controller which maintained $100 \%$ converging simulation rate through $60 \%$ parameter error. The 2 nd order $\mathrm{RC}$ for CPE performed the most poorly of all three RCs.

2) Source Signal Random Period Error: As can be seen in Fig. 11, passive proportional control performed uniformly across all velocity RPE conditions. The 1st order RC and 2nd order RC for RPE perform the best at low error values

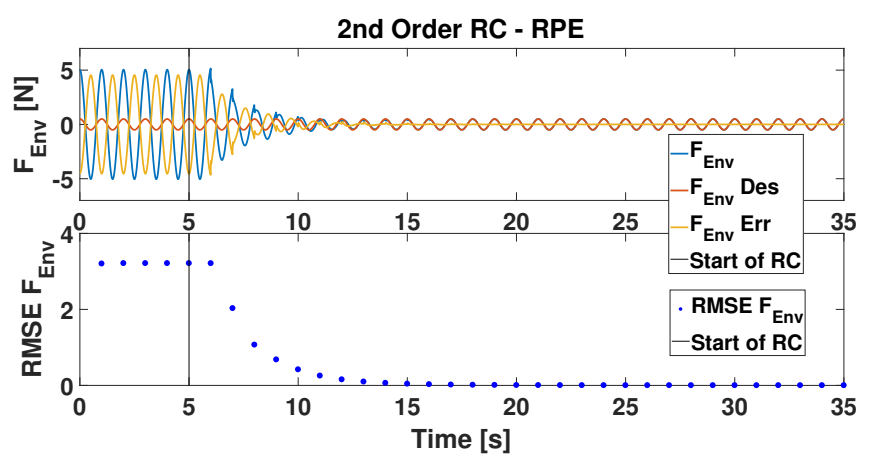

Fig. 7: (Top) example simulation of the plug-in type 2nd order RC, designed for random period error, applied after 5 seconds of passive proportional control. (Bottom) the RMSE of $F_{E n v}$ for each cycle.

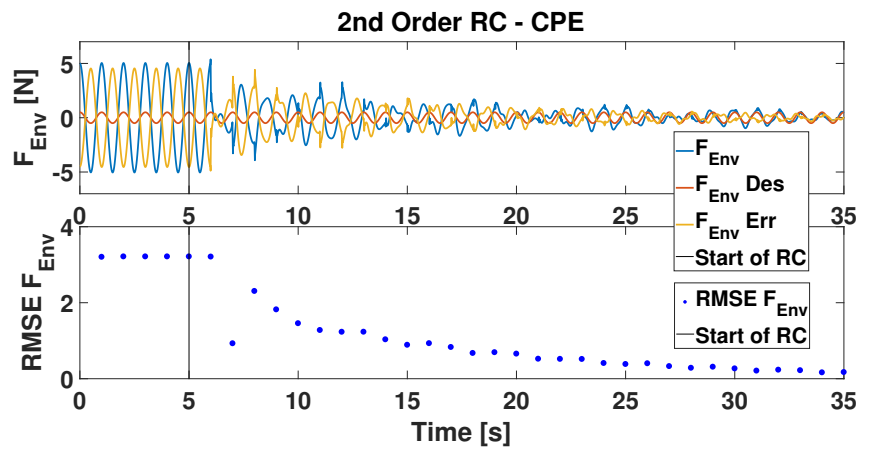

Fig. 8: (Top) example simulation of the plug-in type 2nd order RC, weighted for constant period error, applied after 5 seconds of passive proportional control. (Bottom) the RMSE of $F_{E n v}$ for each cycle.

until $0.30 \mathrm{~Hz}$. At higher error values the $2 \mathrm{nd}$ order $\mathrm{RC}$ for RPE performed better than the 2nd order RC for CPE with both inferior to passive proportional control. The 2nd order $\mathrm{RC}$ for CPE performed with greatest error at all frequency error values.

3) Source Signal Constant Period Error: Shown in Fig. 12; the performance of the four control conditions reflect the sensitivity transfer function plots shown in Fig. 5. The 2nd order RC designed for CPE performed the best at low error values and the 1st order RC performed the best at high error values. The 2 nd order RC designed for RPE performed worse than the other two RCs but better than PPC at low error values.

\section{DISCUSSION AND CONCLUSIONS}

In this paper, we showed that repetitive force control can be successfully applied to a passive impedance controller for the reduction of the end-point impedance and root mean squared force error (RMSFE). We have established that there is a wide range of plant parameter error under which the repetitive controllers (RC) will converge. Furthermore, we have established the effects of constant and random period errors on stability and performance of RC based control using force feedback and concluded that a higher-order RC 


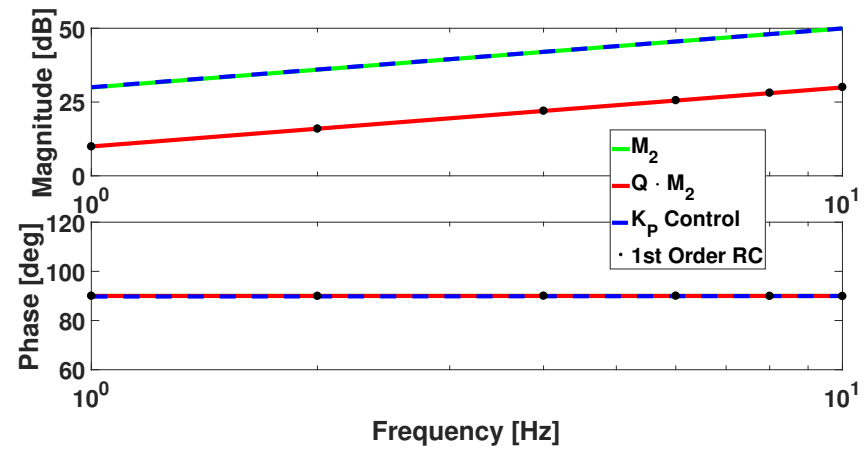

Fig. 9: The (top) magnitudes and (bottom) phases of the theoretical distal mass and fractional distal mass impedances and the measured simulation impedance of the passive controller and repetitive controller.

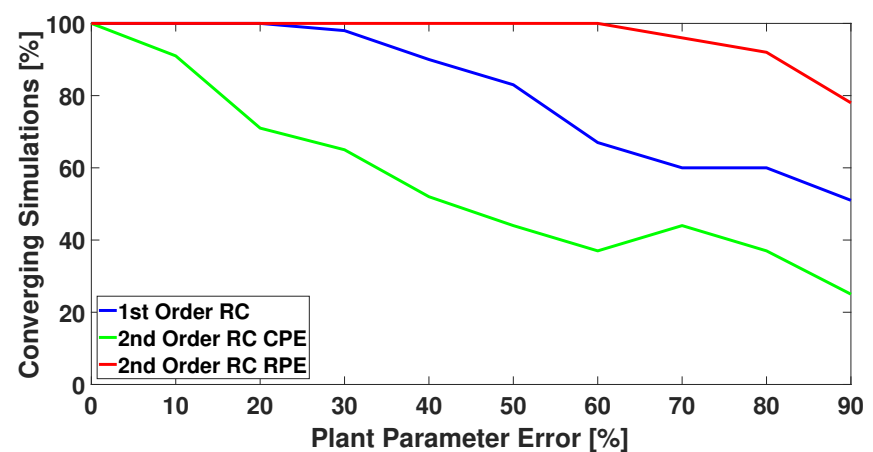

Fig. 10: Rate of convergence of simulations under conditions of parameter error for the three repetitive controllers.

can improve both the robustness to parameter error and the performance of force display under variations in input period.

Under nominal conditions, the 1st order RC successfully reduced the end-point impedance to the target impedance and reduced the RMSFE in the least number of cycles. We found that the 2nd order RC designed for RPE performed the best of all three RCs for the RPE analysis, particularly at high error values. This finding is consistent with previous work in a 3rd order RC analysis [12]. Surprisingly, the 2nd order $\mathrm{RC}$ designed for RPE performed the best in the robustness to plant model error analysis, with $100 \%$ of simulations converging through $60 \%$ parameter error. The 2nd order $\mathrm{RC}$ for CPE did perform the best in the CPE analysis in the region of low error values, which is consistent with previous findings at $0.5 \%$ period time error [10]. However, the 2 nd order RC for CPE performed very poorly in our plant model parameter error and RPE analyses. According to our analyses, we conclude that the optimal form of controller for such an application is a high order RC with positive real fractional weights as in the RPE RC controller. This is due to the fact that we are most concerned with plant model parameter error and random error in signal period, as is practically expected in the implementation of this controller. In regards to $\mathrm{CPE}$, the controller could be reset to a new nominal period, if the CPE exceeds an acceptable value, as might be expected in intentional gait speed changes.

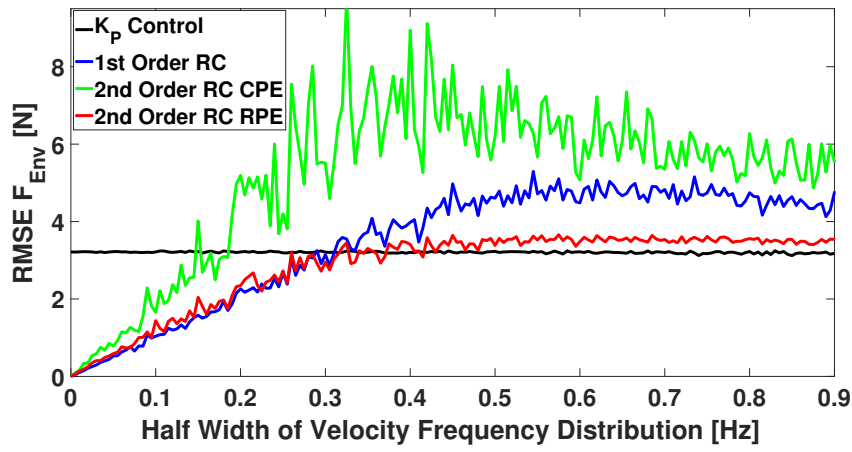

Fig. 11: RMS $F_{E n v_{E r r}}$ for the four force controllers under random error of the period of the environmental velocity signal.

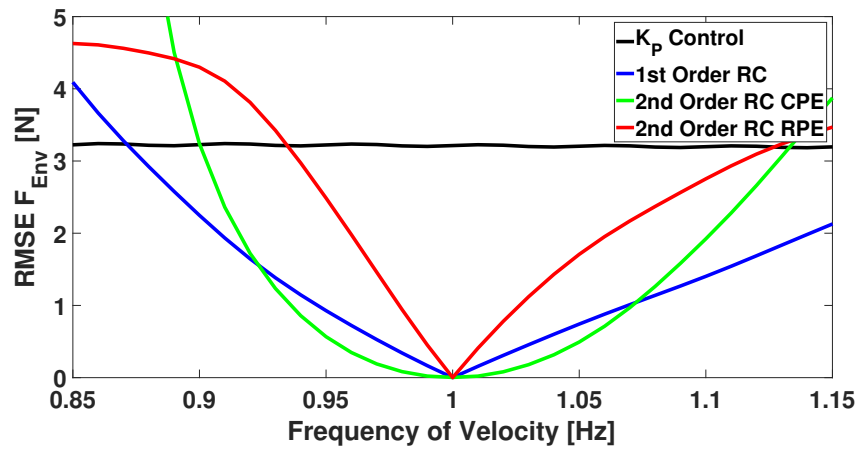

Fig. 12: RMS $F_{E n v_{E r r}}$ for the four force controllers under constant period error of the environmental velocity signal.

Future work will involve the application of higher order (e.g., 5th order) RCs to our force control application in an effort to increase the robustness benefits of higher order RC. We plan to implement the proposed repetitive force controller for impedance reduction to a single degree of freedom joint of a robotic exoskeleton platform and ultimately in a full exoskeleton [18] for purposes of improved transparency.

\section{REFERENCES}

[1] D. Zanotto, T. Lenzi, P. Stegall, and S. K. Agrawal, "Improving transparency of powered exoskeletons using force/torque sensors on the supporting cuffs," in IEEE ICORR, pp. 0-5, 2013.

[2] J. F. Veneman, R. Kruidhof, E. E. Hekman, R. Ekkelenkamp, E. H. Van Asseldonk, and H. Van Der Kooij, "Design and evaluation of the LOPES exoskeleton robot for interactive gait rehabilitation,' IEEE Transactions on Neural Systems and Rehabilitation Engineering, vol. 15, no. 3, pp. 379-386, 2007.

[3] J. E. Colgate and N. Hogan, "Robust control of dynamically interacting systems," Int. J. Control, vol. 48, no. 1, pp. 65-88, 1988.

[4] N. Hogan and S. P. Buerger, "Impedance and Interaction Control 19.1," in Robotics and Hutomation Handbook (T. R. Kurfess, ed.), pp. 19-1 - 19-24, Taylor \& Francis, 2005.

[5] J. Zhang, C. C. Cheah, and S. H. Collins, Torque Control in Legged Locomotion. Elsevier Inc., 1 ed., 2017.

[6] Y. Wang, F. Gao, and F. J. Doyle, "Survey on iterative learning control, repetitive control, and run-to-run control," Journal of Process Control, vol. 19, no. 10, pp. 1589-1600, 2009.

[7] L. Cuiyan, Z. Dongchun, and Z. Xianyi, "A survey of repetitive control," 2004 IEEE/RSJ International Conference on Intelligent Robots and Systems (IROS), vol. 2, pp. 1160-1166, 2004.

[8] F. Pecoraro, C. Mazzà, M. Zok, and A. Cappozzo, "Assessment of level-walking aperiodicity," Journal of NeuroEngineering and Rehabilitation, vol. 3, pp. 1-9, 2006. 
[9] T. Inoue, "Practical repetitive control system design," Proceedings of the IEEE Conference on Decision and Control, vol. 3, pp. 1673-1678, 1990.

[10] M. Steinbuch, "Repetitive control for systems with uncertain periodtime," Automatica, vol. 38, no. 12, pp. 2103-2109, 2002.

[11] R. W. Longman, "On the theory and design of linear repetitive control systems," European Journal of Control, vol. 16, no. 5, pp. 447-496, 2010.

[12] M. Steinbuch, S. Weiland, J. D. Van Eerenbeemt, and T. Singh, "On noise- and period-time sensitivity in high order repetitive control," Proceedings of the IEEE Conference on Decision and Control, vol. 2, pp. 1295-1300, 2004.

[13] H. Guo, R. Longman, and T. Ishihara, "A Design Approach for Insensitivity to Disturbance Period Fluctuations Using Higher Order Repetitive Control," in Preprints of the 19th World Congress The International Federation of Automatic Control, 2014.

[14] H. J. Guo and T. Ishihara, "Some fundamental aspects of second and third order repetitive controllers," 2017 Asian Control Conference, ASCC 2017, vol. 2018-Janua, no. 3, pp. 693-698, 2018.

[15] R. W. Longman, J. W. Yeol, and Y. S. R. Ryu, "Placing the Repetitive Controller Inside or Outside the Feedback Loop: Simultaneously Achieving the Feedback and Repetitive Control Objectives," Advances in astronautical sciences, vol. 127, pp. 1703-1722, 2007.

[16] C. Cosner, G. Anwar, and M. Tomizuka, "Plug in repetitive control for industrial robotic manipulators," IEEE Int Conf Rob Autom, pp. 19701975, 1990.

[17] B. Panomruttanarug and R. W. Longman, "Designing optimized fir repetitive controllers from noisy frequency response data," Advances in the Astronautical Sciences, vol. 127 PART 2, pp. 1723-1742, 2007.

[18] R. L. McGrath and F. Sergi, "Single-stride exposure to pulse torque assistance provided by a robotic exoskeleton at the hip and knee joints," IEEE ICORR, vol. 2019-June, pp. 874-879, 2019. 